\title{
Microbes, Antimicrobials and Resistance: The Battle Goes On
}

\author{
V. C. Kalia
}

Published online: 13 December 2013

(C) Association of Microbiologists of India 2013

Human beings are bestowed with a unique sense of emotions of feeling happy and sad, though not at the same time. We normally link this behavior to worldly experiences. However, a look within and on our body reveals that we are more closely associated with microbes than we are with our family members, friends and society. In fact, human body harbors ten times more microbial cells than their own. When we are happy and healthy, we do not notice the presence of microbes, who are working silently for our well being. We take notice of their presence only when they make noise and cause disease. Skin from different parts of our body harbors microbiota with Corynebacteria, Propionibacteria, Staphylococcus, Proteobacteria, Bacteroidetes as dominant members of this community. Inside the human body, the gut microbiota is responsible for our physique. We look physically fit when Firmicutes and Bacteroidetes constitute $44-48 \%$ of the total gut microbiota. In contrast, we grow obese, when these two bacteria attain dominance at $82-86 \%$ of the total population, where Firmicutes are responsible for high fat intake and Bacteroidetes for high carbohydrate intake. In addition, the sub-dominant bacterial groups are microbes belonging to Actinobacteria, Fusobacteria, and Verrucomicrobia. Beyond these microbes, the human body is also frequented by visiting bacteria. Here, Pseudomonas enjoys staying in the body and cause infectious disease, whereas Vibrios use the human body to multiply and leave them at will through

V. C. Kalia $(\bowtie)$

Microbial Biotechnology and Genomics, CSIR-Institute of Genomics and Integrative Biology, Delhi University Campus, Mall Road, New Delhi 110007, India

e-mail: vckalia@igib.res.in; vc_kalia@yahoo.co.in

URL: http://scholar.google.co.in/citations?hl=en\&user=XaUw-

VIAAAAJ diarrheal manifestations. A few others among the category of being notorious and most feared by human beings are: Staphylococcus aureus, Clostridium spp., Yersinia enterocolitica, Streptococcus mutans, Streptococcus pneumoniae, etc. These bacterial infections are responsible for high mortality and morbidity in man and are a major cause of worry for Health Departments around the world. The discovery of antibiotics brought relief to many. Sir Alexander Fleming was honoured with the Nobel prize for the discovery of the wonderful antibiotic-penicillin. Antibiotics have been realized to be the most effective drug for treating infectious diseases. However, microbes have been constantly evolving and becoming resistant to antibiotics: penicillin resistant Staphylococcus in 1940, tetracycline resistant Shigella emerged in the second half of the twentieth century, and erythromycin resistant Streptococcus in the late 1960s. During the last six decades, many antibiotics were found to have become ineffective within a few years of their launch, as bacteria were found to have developed resistance to them. It is now believed that microbes have the potential arsenal necessary to counter almost all conceivable antibiotics. So much so that pharmaceutical industry is finding it counter productive to invest in this area [1]. It has pushed researchers to the edge and forced them to have a re-look on the ways to attack microbes. Recent studies have revealed that more than $80 \%$ of the infectious diseases are caused by biofilmforming bacteria. Bacteria within the biofilm are upto 1,000 times more resistant to antibiotics than their planktonic counterparts. Studies on the elucidation of mechanism of biofilm formation have led to the finding that it is regulated through genes which get expressed in a cell density dependent manner. The phenomenon is now termed as quorum sensing, QS [2]. As a single cell or low cell density, bacteria do not form biofilm, but at high cell density, 
this pathogenic feature gets expressed making bacteria behave like multicellular organisms. While bacteria are not forming biofilm, they use this phenomenon for their growth, development, sporulation, virulence, toxins, cell division, motility, bioluminescence, antibiotic production, etc. [2]. Apart from Health, the other departments which dread these pathogens are: (i) fishery departments, who worry about-Aeromonas, Vibrio and Yersinia spp., (ii) biofouling: biofilms formed on reverse osmosis membranes used for producing drinking water and reclamation of wastewater. Incidentally, it is also responsible for the rapid deterioration of archaeological buildings and monuments. QS operates through signal molecules. Efforts to inhibit pathogenicity through quenching these signal molecules have been quite fruitful [2, 3]. These quorum sensing inhibitors (QSIs) can be used to develop drugs to target QS mediated gene expression. QSIs have been primarily targeting biosynthesis and degradation of signal molecules and their receptors. QSI from prokaryotes, animal and plant origin, have been widely reported [2-6]. Synthetic QSIs have more variety, efficacy and efficiency in comparison to naturally occurring ones [3]. Synergism between antibiotics and QSIs have been effective in enhancing the action of antibiotics, albeit in experimental stages only. The chances of successful use of QSIs seem higher since their usage does not affect growth of the organism and selectively repress expression of genes responsible for their virulent behavior [2, 3]. Now we are at the crossroad, where questions are being raised if QSIs will meet the same fate as that of antibiotics. Will bacteria develop resistance to QSIs? [7]. Are we prepared to mutely witness yet another Never Ending battle?

\section{References}

1. Kalia VC, Rani A, Lal S, Cheema S, Raut CP (2007) Combing databases reveals potential antibiotic producers. Expert Opin Drug Discov 2:211-224. doi:10.1517/17460441.2.2.211

2. Kalia VC, Purohit HJ (2011) Quenching the quorum sensing system: potential antibacterial drug targets. Crit Rev Microbiol 37:121-140. doi:10.3109/1040841X.2010.532479

3. Kalia VC (2013) Quorum sensing inhibitors: an overview. Biotechnol Adv 31:224-245. doi:10.1016/j.biotechadv.2012.10. 004

4. Kalia VC, Raju SC, Purohit HJ (2011) Genomic analysis reveals versatile organisms for quorum quenching enzymes: acyl-homoserine lactone-acylase and -lactonase. Open Microbiol J 5:1-13. doi: $10.2174 / 1874285801105010001$

5. Huma N, Shankar P, Kushwah J, Bhushan A, Joshi J, Mukherjee T, Raju SC, Purohit HJ, Kalia VC (2011) Diversity and polymorphism in AHL-lactonase gene (aiiA) of Bacillus. J Microbiol Biotechnol 21:1001-1011. doi:10.4014/jmb.1105.05056

6. Kumar P, Patel SKS, Lee JK, Kalia VC (2013) Extending the limits of Bacillus for novel biotechnological applications. Biotechnol Adv 31:1543-1561. doi:10.1016/j.biotechadv.2013.08.007

7. Kalia VC, Wood TK, Kumar P (2013) Evolution of resistance to quorum-sensing inhibitors. Microb Ecol. doi:10.1007/s00248-0130316-y 